\title{
Phylogenetic Relationships of Salmonella typhi and Salmonella typhimurium Based on 16S rRNA Sequence Analysis
}

\author{
HERNAN R. CHANG, ${ }^{1 *}$ L. H. LOO, ${ }^{1}$ K. JEYASEELAN, ${ }^{2}$ L. EARNEST, ${ }^{2}$ AND E. STACKEBRANDT ${ }^{3}$ \\ Department of Microbiology ${ }^{1}$ and Department of Biochemistry, ${ }^{2}$ Faculty of Medicine, National University \\ of Singapore, Singapore 0511, Republic of Singapore, and DSMZ-Deutsche Sammlung von \\ Mikroorganismen und Zellkulturen GmbH, 38124 Braunschweig, Germany ${ }^{3}$
}

\begin{abstract}
The 16S rRNA gene sequences of Salmonella typhi and Salmonella typhimurium were amplified by PCR, cloned, and sequenced. These sequences were analyzed by comparison with reference organisms from the family Enterobacteriaceae. Both $S$. typhi and $S$. typhimurium belong to the gamma subdivision of the class Proteobacteria.
\end{abstract}

The genus Salmonella is a genus of the family Enterobacteriaceae. The following two different classification systems have been validly proposed for species of the genus Salmonella: (i) the two-species system, with Salmonella enterica and Salmonella bongori; and (ii) the five-species system, with $S$. bongori, Salmonella choleraesuis, Salmonella enteritidis, Salmonella typhi, and Salmonella typhimurium. In this communication we follow the five-species classification. All species share a high degree of DNA homology (2). By using cross-absorption and antiserum reactions to differentiate $O$ (somatic or polysaccharide) and $\mathrm{H}$ (flagellar) antigens, workers have defined over 2,000 Salmonella serotypes (18). Some serotypes have a marked host specificity, and some others can infect many animal species besides humans and can produce invasive disease, such as enteric fever. For instance, $S$. typhi causes enteric (typhoid) fever and is only pathogenic in humans, whereas $S$. typhimurium causes gastroenteritis and is pathogenic for several mammalian species. Bacteriophage typing has been used to differentiate $S$. typhi within serotypes (6). Despite their different sensitivities to specific bacteriophages, these bacteria appear to be clonal, as determined by multilocus enzyme electrophoresis and DNA hybridization analysis (11). In order to gain insight into the phylogenetic relationships of both $S$. typhi and $S$. typhimurium, we cloned and sequenced their $16 \mathrm{~S}$ ribosomal DNAs (rDNAs) (5) by using a PCR approach.

Cloning and sequencing of 16S rRNA genes. $S$. typhi ATCC 19430 was obtained from the American Type Culture Collection (Rockville, Md.), and S. typhimurium NCTC 8391 was obtained from the Culture Collection of the Department of Microbiology, National University of Singapore. Single colonies of these strains were grown in liquid media, and their genomic DNAs were extracted by lysis with lysozyme and sodium dodecyl sulfate followed by isolation with $N$-cetyl- $N, N, N$ trimethylammonium bromide (Merck, Darmstadt, Germany) and precipitation with ethanol (16). Each purified DNA was subjected to PCR with universal primers containing flanking EcoRI sites (17). The amplified 16S rDNAs were purified and ligated to the pT7Blue T-vector (Novagen, Inc., Madison, Wis.) according to the manufacturer's instructions and were transformed by electroporation into competent Escherichia coli. After selection of recombinant colonies, isolated colonies

\footnotetext{
* Corresponding author. Present address: Laboratory of Nutrition/ Infection, CRI-3, Department of Medicine, Beth Israel Deaconess Medical Center, West Campus/Harvard Medical School, 194 Pilgrim Road, Boston, MA 02115. Phone: (617) 632-0132. Fax: (617) 632-0204. E-mail: rochang@west.bidmc.harvard.edu.
}

were grown, and their plasmid DNAs were extracted and purified (14). Each purified plasmid DNA was amplified by using a Taq DyeDeoxy terminator cycle sequencing kit in a DNA thermal cycler (Perkin-Elmer Cetus, Norwalk, Conn.) and was analyzed by using a model $373 \mathrm{~A}$ automated DNA sequencer (Applied Biosystems, Inc., Foster City, Calif.) and a set of sequencing primers. In addition, following digestion of clones with EcoRI (New England Biolabs, Inc., Beverly, Mass.) and subcloning into pUC19 plasmids, we sequenced both strands of three $S$. typhi subclones and two $S$. typhimurium subclones.

Phylogenetic analysis. Pairwise evolutionary distances were computed from the $16 \mathrm{~S}$ rDNA similarity values (data not shown) by using the correction of Jukes and Cantor (7). The distance matrix method of De Soete (3) and neighbor-joining analysis $(4,13)$ were used to reconstruct phylogenetic trees from the distance matrix. Bootstrap values, calculated for 300 trees, were generated by using the algorithms Nhboot and NJFind. Almost complete $16 \mathrm{~S}$ rDNA sequences were determined for S. typhi ATCC 19430 and S. typhimurium NCTC 8391; these sequences comprised $99.2 \%$ of the Escherichia coli $16 \mathrm{~S}$ rDNA sequences (1). The two sequences were aligned with the homologous sequences of members of the family Enterobacteriaceae for which almost complete $16 \mathrm{~S}$ rDNA sequences had been deposited in the EMBL or Ribosomal Database Project (10) (Fig. 1), and similarity values were determined. The sequences of the two Salmonella strains show $99.7 \%$ similarity. The five differences are found at positions 572, 609, 1136,1137 , and 1269 . The similarity values for the two Salmonella strains and other members of the Enterobacteriaceae range between 97.6 and $91.7 \%$. The phylogenetic trees, generated on the basis of corrected similarity values (7), were identical in that the two Salmonella strains were placed in a phylogenetic cluster that contains Enterobacter cloacae, Escherichia coli and Citrobacter freundii (96.3 to $97.6 \%$ similarity). Pantoea agglomerans (about $95 \%$ similarity), Serratia marcescens $(95.5$ to $96 \%$ similarity), and Erwinia carotovora (95.2 to $95.7 \%$ similarity) were moderately related to the Escherichia coli cluster, while the other strains belonging to the Enterobacteriaceae included in this study formed a separate subline of descent. A phylogenetic dendrogram is shown in Fig. 1.

Analysis of the two Salmonella sequences with unpublished short $16 \mathrm{~S}$ rDNA stretches of about 300 nucleotides (between positions 300 and 600 ) from $S$. typhimurium K-12, S. typhi, $S$. choleraesuis, S. enteritidis, and "Salmonella dublin" which are deposited in the EMBL revealed $100 \%$ sequence similarity (data not shown). The nucleotide sequence of this stretch is so conserved that even the $16 \mathrm{~S}$ rDNAs of Salmonella species and 


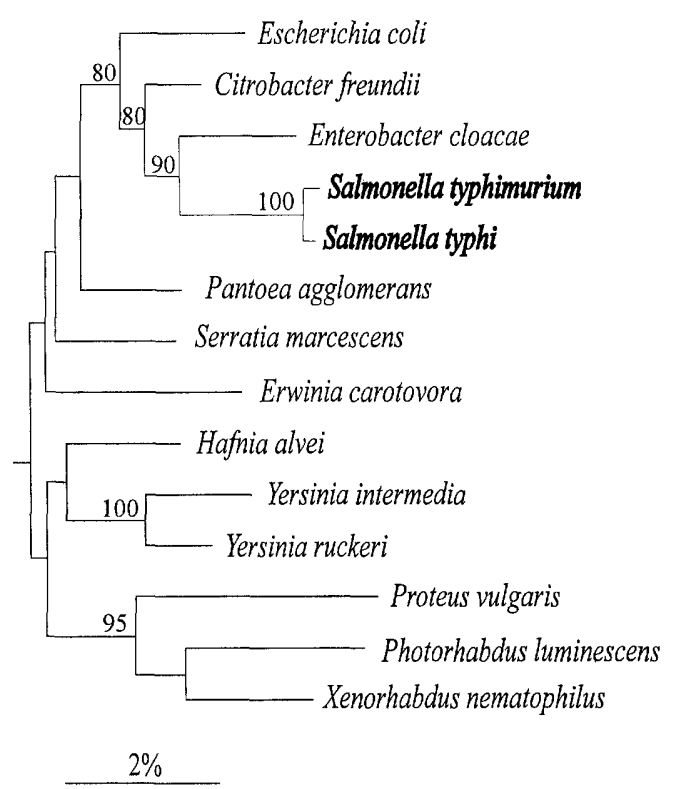

FIG. 1. Phylogenetic dendrogram based on distance matrix analysis of $16 \mathrm{~S}$ rRNA gene sequences from $S$. typhi and $S$. typhimurium strains sequenced in this work and reference strains. Reference sequences of the following organisms were obtained from EMBL: Escherichia coli ATCC 25922 (accession no. X80724), Photobacter luminescens DSM 3368 (X82248), Proteus vulgaris IFAM 1731 (X07652), Xenorhabdus nematophilus DSM 3370 (X82251), Yersinia intermedia ER-3854 (X75279), and Yersinia ruckeri ATCC 29473 (X75275). Reference sequences of the following organisms were obtained from the Ribosomal Database Project (10), in which no strain designations are indicated for the species: Citrobacter freundii (accession no. M59291), Erwinia carotovora (M59149), Serratia marcescens (M59160), Pantoea agglomerans, Hafnia alvei (M59155), and Plesiomonas shigelloides (M59159). The sequence of Enterobacter cloacae DSM 30054 was kindly provided by F. A. Rainey.

those of the Escherichia coli strains are either absolutely identical or differ only in one or two nucleotides. Consequently, analysis of this stretch does not allow discrimination of phylogenetic relationships.

Previous DNA-DNA reassociation data $(2,8,9,15)$ and the 16S rRNA gene sequence similarities correlate very well: (i) both methods place the genus Salmonella in the family Enterobacteriaceae; (ii) the close $16 \mathrm{~S}$ rDNA relationship of Salmonella species to members of the genera Escherichia and Citrobacter, shown previously by DNA-DNA reassociation to be about $45 \%$ (2), is confirmed; and (iii) the results of both methods indicate that the genera Erwinia, Pantoea, and Serratia are less closely related to the genera Salmonella, Escherichia, and Citrobacter and that the genera Yersinia, Hafnia, Proteus, Xenorhabdus, and Photorhabdus are even more remotely related. The genus Salmonella contains five validly described species, which are highly related as determined by DNA-DNA reassociation. The high degree of DNA hybridization similarity for $S$. typhi and $S$. typhimurium (84\%) (2) was confirmed by a high 16S rDNA similarity value $(99.7 \%)$.

Probes developed for the 23S rRNA of Salmonella species could be used to discriminate between two groups of Salmo- nella species, one containing $S$. typhimurium, S. typhi, S. enteritidis, and $S$. choleraesuis and the other containing $S$. bongor (12). These results confirmed those obtained by Reeves et al. (11) by multilocus enzyme electrophoresis. The $16 \mathrm{~S}$ rDNA sequences obtained in this work may be used to improve diagnostic tools for members of the genus Salmonella based on molecular genetics.

Nucleotide sequence accession numbers. The nucleotide sequences determined in this study have been deposited in the EMBL database (Cambridge, United Kingdom) under the following accession numbers: S. typhi ATCC $19430^{\mathrm{T}}$, Z47544; and S. typhimurium NCTC 8391, Z49264.

This work was supported by grant RP390373 from the National University of Singapore.

We are grateful to S. H. Chan for his interest and encouragement.

\section{REFERENCES}

1. Brosius, J., M. L. Palmer, J. P. Kennedy, and H. P. Noller. 1978. Complete nucleotide sequence of a $16 \mathrm{~S}$ ribosomal RNA gene from Escherichia coli. Proc. Natl. Acad. Sci. USA 75:4801-4805.

2. Crosa, J. H., D. J. Brenner, W. H. Ewing, and S. Falkow. 1973. Molecular relationships among the Salmonelleae. J. Bacteriol. 115:307-315.

3. De Soete, G. 1983. A least squares algorithm for fitting additive trees to proximity data. Psychometrika 48:621-626.

4. Felsenstein, J. 1993. PHYLIP (phylogeny inference package), version 3.5c. J. Felsenstein, University of Washington, Seattle.

5. Fox, G. E., E. Stackebrandt, R. B. Hespell, J. Gibson, J. Maniloff, T. A. Dyer, R. S. Wolfe, W. E. Balch, R. Tanner, L. Magrum, L. B. Zablen, R. Blakemore, R. Gupta, L. Bonen, B. J. Lewis, D. A. Stahl, K. R. Luehrsen, K. N. Chen, and C. R. Woese. 1980. The phylogeny of prokaryotes. Science 209:457-463.

6. Gershman, M. 1977. Single phage-typing set for differentiating salmonellae. J. Clin. Microbiol. 5:302-314.

7. Jukes, T. H., and C. R. Cantor. 1969. Evolution of protein molecules, p. 21-132. In $\mathrm{H}$. N. Munro (ed.), Mammalian protein metabolism. Academic Press, Inc., New York, N.Y.

8. Le Minor, L., M. Y. Popoff, B. Laurent, and D. Hermant. 1986. Individualisation d'une septième sous-espèce de Salmonella: $S$. choleraesuis subsp. indica subsp. nov. Ann. Inst. Pasteur (Paris) 137B:211-217.

9. Le Minor, L., R. Rohde, and J. Taylor. 1970. Nomenclature des Salmonella. Ann. Inst. Pasteur 119:206-210.

10. Maidak, B. L., N. Larsen, M. J. McCaughey, R. Overbeek, G. J. Olsen, K. Fogel, J. Blandy, and C. R. Woese. 1994. The Ribosomal Database Project. Nucleic Acids Res. 22:3485-3487.

11. Reeves, M. W., G. M. Evins, A. A. Heiba, B. D. Plikaytis, and J. J. Farmer III. 1989. Clonal nature of Salmonella typhi and its genetic relatedness to other salmonellae as shown by multilocus enzyme electrophoresis, and proposal of Salmonella bongori comb. nov. J. Clin. Microbiol. 27:313-320.

12. Rönner, S. G. E., and E. Stackebrandt. 1994. Development of 23S rDNAoligonucleotide probes for the identification of Salmonella species. Syst. Appl. Microbiol. 17:257-264.

13. Saitou, N., and M. Nei. 1987. The neighbor-joining method: a new method for reconstructing phylogenetic trees. Mol. Biol. Evol, 4:406-425.

14. Sambrook, J., E. F. Fritsch, and T. Maniatis. 1989. Molecular cloning: a laboratory manual, 2nd ed. Cold Spring Harbor Laboratory, Cold Spring Harbor, N.Y.

15. Stoleru, G. H., L. Le Minor, and A. M. Lheritier. 1993. Polynucleotide sequence divergence among strains of Salmonella sub-genus IV and closely related organisms. Ann. Microbiol. (Paris) 127A:477-486.

16. Towner, P. 1992. Purification of DNA, p. 47-55. In T. A. Brown (ed.) Essential molecular biology, a practical approach, vol. 1. IRL Press at Oxford University Press, Oxford, United Kingdom.

17. Weisberg, W. G., S. M. Barns, D. A. Pelletier, and D. J. Lane. 1991. 16S ribosomal DNA amplification for phylogenetic analysis. J. Bacteriol. 173: 697-703.

18. World Health Organization. 1980. Center for Reference and Research on Salmonella antigenic formulae of the Salmonella. WHO International Salmonella Center, Pasteur Institute, Paris, France. 